logical aspects of investigation of population contingents irradiated in the accident on Chernobyl nuclear power plant. Vestnik AMN SSSR $1991 ; 8: 16-20$

3. Shubik V: Immunological investigations in radiation hygiene. Energoatomizdat, Moscow 1987, p 147.

4. Maltezev VN: Quantitative dependencies in radiation immunology. Energoatomizdal, Moscow $1983, \mathrm{p} 88$.

5. Yamada $Y$, Nerishi S, Ishimaru T: Effects of atomic bomb radiation on the differentiation of B-lymphocytes and on the function of con-
Canavalin A-induced suppressor T-lymphocytes. Radiation Research 1985;101:351-355.

6. Galitskasya NN, Hmelevskaya L, Zhuk G: T and $B$ cellular immunity in children exposed to ionizing radiation. Radiobiologia (Moscow) 1990;30:562.

7. Korobko IV Titov LP: Interferon system in children of school age living in Bragin and Cherikov districts of Byelorussia. Zdravoohr Belonussii (Minsk) 1990;6:48.

8. Results of estimation of the medical consequences of Chemobyl NPP accident. Theses of communications on Republican scientific and practical conference. Kjev 1991, p 255.

9. Beverley PCL: Clinical and biological studies with monoclonal antibodies. In: Monoclomal antibodies. Beverely PCL (ed). Edinburgh - London Melbourne New York: Churchill Livingstone 1986, pp 247-270.

10. Kyosumi S, Nakamura N, Yakoda $M$, et al: Detection of somatic mutations at the glycophorin A locus in erythrocytes of atomic bomb survivors using a single beam flow sorter. Cancer Research 1989;49:581-588.

\title{
Medical-Evacuation Characteristics of Persons Affected During Earthquakes
}

\section{S.F. Goncharov, $M D,{ }^{I}$ V.I. Chadov, $M D^{2}$}

1. Director, All-Russian Center for Disaster Medicine, Moscow, Russia

2. Deputy Director, All-Russian Center for Disaster Medicine, Moscow, Russia

The notion of medical-evacuation characteristics (MEC) of people affected by earthquakes is a broad one that includes estimating the needs of the affected people with regard to different medical measures. The data needed include dates, results, places of treatment, and evacuation features. In spite of the general acknowledgment of the importance of such estimations in determining, not only the situation in the seismic center, but also the organization of the medicalevacuation ensurance (MEE), this problem has not been studied scientifically in a comprehensive manner.

Even the volumes of material about the earthquakes in Armenia (1988) and Loma Prieta (1989) do not provide an integration of the MEC of the affected people. This can be explained not only by the severe medical after-effects of each earthquake and problems with the established organization of medicalevacuation ensurance, but also by some subjective reasons. These include imperfect methods of studying earthquake consequences and the accounting systems used.

In accordance with the organization of the MEE adopted by the All-Russia Disaster Medicine Service, the MEC investigation is grounded in assessing the needs of the affected person for the most labor-intensive measures in the acute response period and for later more skilled medical aid. These include complex anti-shock therapy; surgical interventions, including urgent hospitalization of untransportable patients; immobilization; Novocain block, etc. The MEE also examines the distribution according to the medical institutions where the patients are treated (headneck-spine; thoracoabdominal, trauma, etc.).

These results are of scientific and practical importance.

\section{Psychological Monitoring}

\section{Strategies for Comprehensive Monitoring of Mid-and Long-Term Effects of Disasters Gloria R. Leon, PhD}

Professor, Department of Psychology, University of Minnesota, Minneapolis, Minnesota USA

A conceptual model is presented of radiation exposure effects on psychological and physical health. This model illustrates the breadth of impact of a technological disaster, and the various pathways through which the disaster affects health. In addition to radiationinduced physical health effects, radiation exposure also can result in significant psychological stress. Over time and through a series of steps, this stress may cause clinical symptoms and physical diseases.

Psychological stress is proposed to have a direct effect on personal, family, and community disruption, as well as perceived uncontrollability over one's life and distrust of government. These disruptive influences, in turn, cause family dysfunction, poor social support, other traumatic stresses, economic problems, and accident-proneness. Psychological stress also is proposed to have a direct influence on concerns about the effects of radiation on health, resulting in sensitivity to physical symptoms and complaints. Both family and social consequences of stress and symptom sensitivity may lead to psychological dysfunction, including post-traumatic stress disorder, anxiety, depression, and maladaptive coping strategies. These manifestations of psychological dysfunction can result in increased alcohol and cigarette use, with a direct effect on clinical symptoms such as elevated blood pressure and other cardiovascular problems. Clinical symptoms, in turn, have a direct effect on physical disease, including stress-related disorders and exacerbation of chronic diseases.

Suggested assessment tools and criteria for monitoring psychosocial and gen- 
eral health effects in large populations after large-scale disasters are described. The use of short, reliable psychological measures that assess a broad spectrum of dysfunction is imperative in screening large numbers of people. Specific areas to evaluate in which the stress effects are manifested include post-traumatic stress disorder, somatic symptoms and complaints, and mental disorder diagnosis and treatment. Indices of family dismup- tion can be obtained through information on the intactness of the family unit and possible spouse and child abuse. Lifestyle indices of stress and community disruption include presence of alcohol and other substance use, cigarette smoking, accidents, and suicides. Health monitoring also consists of the collection of morbidity and mortality data from hospital and clinic records, with a particular interest in cardiovascular and gastrointestinal disorders as manifestations of stress. Data on relocation, unemployment, personal and property crime victimization, and distrust of the government assess the impact of a disaster on the social structure of the community. Through examination of a disaster with foci ranging from personal to societal levels, a more comprehensive view of mid- and long-term effects can be obtained.

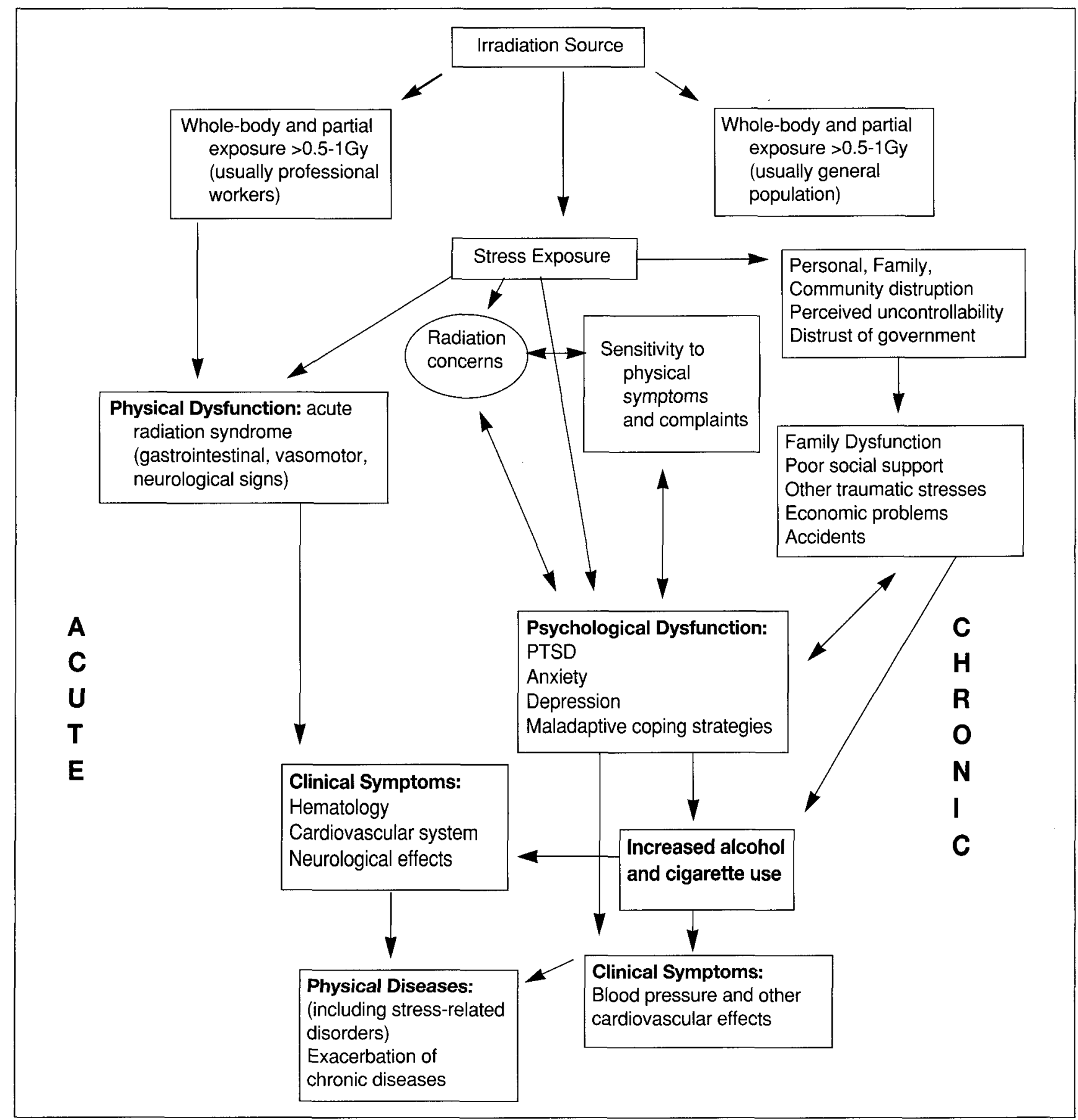

Conceptual model of radiation exposure effects on psychological and physical health 